Simulation and Analysis of Large-Scale Compton Imaging Detectors

H. A. Manini, D. J. Lange, D. M. Wright

December 27, 2006 
This document was prepared as an account of work sponsored by an agency of the United States Government. Neither the United States Government nor the University of California nor any of their employees, makes any warranty, express or implied, or assumes any legal liability or responsibility for the accuracy, completeness, or usefulness of any information, apparatus, product, or process disclosed, or represents that its use would not infringe privately owned rights. Reference herein to any specific commercial product, process, or service by trade name, trademark, manufacturer, or otherwise, does not necessarily constitute or imply its endorsement, recommendation, or favoring by the United States Government or the University of California. The views and opinions of authors expressed herein do not necessarily state or reflect those of the United States Government or the University of California, and shall not be used for advertising or product endorsement purposes.

This work was performed under the auspices of the U.S. Department of Energy by University of California, Lawrence Livermore National Laboratory under Contract W-7405-Eng-48. 


\title{
Simulation and Analysis of Large-Scale Compton Imaging Detectors
}

\author{
Hugh Manini, David Lange, Douglas Wright \\ High Energy Physics Group \\ N Division \\ Lawrence Livermore National Laboratory
}

December 18, 2006

\begin{abstract}
We perform simulations of two types of large-scale Compton imaging detectors. The first type uses silicon and germanium detector crystals, and the second type uses silicon and CdZnTe (CZT) detector crystals. The simulations use realistic detector geometry and parameters. We analyze the performance of each type of detector, and we present results using receiver operating characteristics (ROC) curves.
\end{abstract}

\section{Detector simulations}

We perform full physics simulations using GEANT4 of the Si-Ge and Si-CZT detectors. Each large-scale Compton imaging detector system consists of a planar $16 \times 16$ array of detector modules. The Si-Ge $16 \times 16$ array has a total detector area of $3.33 \mathrm{~m}^{2}$, and the Si-CZT $16 \times 16$ array has a total detector area of $3.15 \mathrm{~m}^{2}$.

Each GEANT4 simulation is run once for each specific detector geometry. Source photons from a point source are generated at a specific energy and tracked through the detector volumes. The positions and energies for each interaction are stored for further analysis. We then "digitize" the exact information and introduce detector resolution effects, so that the resulting simulated output mimics the realistic detector response. The $x$ and $y$ coordinates of the interactions within the active detector crystals are pixelized. The $\mathrm{Si}-\mathrm{Ge}$ detector has detection strips on each detector crystal which are $2 \mathrm{~mm}$ wide. This produces pixels in the $x y$-plane that are each $2 \mathrm{~mm} \times 2 \mathrm{~mm}$ in size (the $x y$-plane is parallel to the front surface of each detector crystal). The Si-CZT detector has pixels that are each $1 \mathrm{~mm} \times 1 \mathrm{~mm}$ in size. When more than one interaction occurs within a pixel, the interactions and deposited energies are combined. The detected energy distribution is determined by smearing individual energy deposits using a Gaussian probability distribution with a specified FWHM at each energy, to model the energy resolution. The interaction depth within the detector is represented by the $z$ coordinate, and we model the $z$ resolution by smearing the exact $z$ coordinate of each interaction using a Gaussian probability distribution with a FWHM of $0.5 \mathrm{~mm}$, for each type of detector. 


\subsection{Si-Ge simulated detector geometry}

The simulated detector geometry for each $\mathrm{Si}-\mathrm{Ge}$ module includes the detector crystals and the module cryostat. The simulated detector geometry for an Si-Ge module is shown in Figure 1. Each Si-Ge module contains two Si detector crystals that are each $64 \mathrm{~mm} \times 64 \mathrm{~mm} \times 10 \mathrm{~mm}$ in size, and two Ge detector crystals that are each circular disks that are $84 \mathrm{~mm}$ in diameter and $15 \mathrm{~mm}$ thick. The active volume of each Ge detector crystal is the volume of the Ge disk within a concentrically inscribed box which is $76 \mathrm{~mm} \times 76 \mathrm{~mm} \times 15 \mathrm{~mm}$ in size. The Si detector crystals are very useful for providing imaging capability for photon energies below $150 \mathrm{keV}$. The separation distance between each of the detector crystals is $8 \mathrm{~mm}$. The module cryostat completely encloses the detector crystals. The cryostat walls are rectangular in shape, and made of aluminum. The front and back cryostat walls are both $2 \mathrm{~mm}$ thick. All other cryostat walls are $5 \mathrm{~mm}$ thick. The distance between the front cryostat wall and the front Si detector is $8 \mathrm{~mm}$, and the distance between the back cryostat wall and the second Ge detector is $8 \mathrm{~mm}$. The closest distance between the edges of the Ge detectors and the cryostat walls is $10 \mathrm{~mm}$. The large-scale Si-Ge detector system consisting of a $16 \times 16$ array of detector modules has a total detector area of $3.33 \mathrm{~m}^{2}$. The simulated detector geometry for the $16 \times 16$ array of detector modules is shown in Figure 3. In the planar $16 \times 16$ array of detector modules, there is a $1 \mathrm{~mm}$ gap between the edges of each detector module. Table 1 shows the area contributions for various detector components for the Si-Ge $16 \times 16$ array.

\subsection{Si-CZT simulated detector geometry}

The simulated detector geometry for each Si-CZT module includes the detector crystals and the aluminum module walls. The simulated detector geometry for an Si-CZT module is shown in Figure 2. Each SiCZT module contains $10 \mathrm{Si}$ detector crystals, and $72 \mathrm{CZT}$ detector crystals. Each Si detector crystal is $70 \mathrm{~mm} \times 70 \mathrm{~mm} \times 2 \mathrm{~mm}$ in size, with an amount of inactive $\mathrm{Si}$ around the side edges which is $3 \mathrm{~mm}$ wide. This leaves an active portion for each Si crystal which is $64 \mathrm{~mm} \times 64 \mathrm{~mm} \times 2 \mathrm{~mm}$ in size. The Si detector crystals are very useful for providing imaging capability for photon energies below $150 \mathrm{keV}$. The separation distance between the Si detector crystals is $5 \mathrm{~mm}$. The Si-CZT detector operates at room temperature, so it must use thin Si detector crystals, because thick Si detector crystals require cooling. Behind the Si detector crystals inside a detector module are two $6 \times 6$ arrays of CZT detector crystals. Each CZT detector crystal is $15 \mathrm{~mm} \times 15 \mathrm{~mm} \times 10 \mathrm{~mm}$ in size, with a thin layer of inactive CZT around the side edges which is $0.1 \mathrm{~mm}$ thick. This leaves an active portion for each CZT crystal which is $14.8 \mathrm{~mm} \times 14.8 \mathrm{~mm} \times 10 \mathrm{~mm}$ in size. The separation distance between each CZT detector crystal in an array is $1 \mathrm{~mm}$. The separation distance between the last Si detector and the first $6 \times 6$ array of CZT crystals is $10 \mathrm{~mm}$, and the separation distance between the two $6 \times 6$ arrays of CZT crystals is $10 \mathrm{~mm}$. The walls of the module completely enclose the detector crystals. The module walls are rectangular in shape, and made of aluminum. Each module wall is $2 \mathrm{~mm}$ thick. The separation distance between the first Si detector and the front module wall is $5 \mathrm{~mm}$, and the separation distance between the second CZT array and the back module wall is $5 \mathrm{~mm}$. The closest distance between the edge of each CZT array and the module walls is $6 \mathrm{~mm}$. The large-scale Si-CZT detector system consisting of a $16 \times 16$ array of detector modules has a total detector area of $3.15 \mathrm{~m}^{2}$. The simulated detector geometry for the $16 \times 16$ array of detector modules is shown in Figure 3 . In the planar $16 \times 16$ array of detector modules, there is a $1 \mathrm{~mm}$ gap between the edges of each detector module. Table 1 shows the area contributions for various detector components for the Si-CZT 16×16 array. 


\section{Si-Ge module}

(side view)

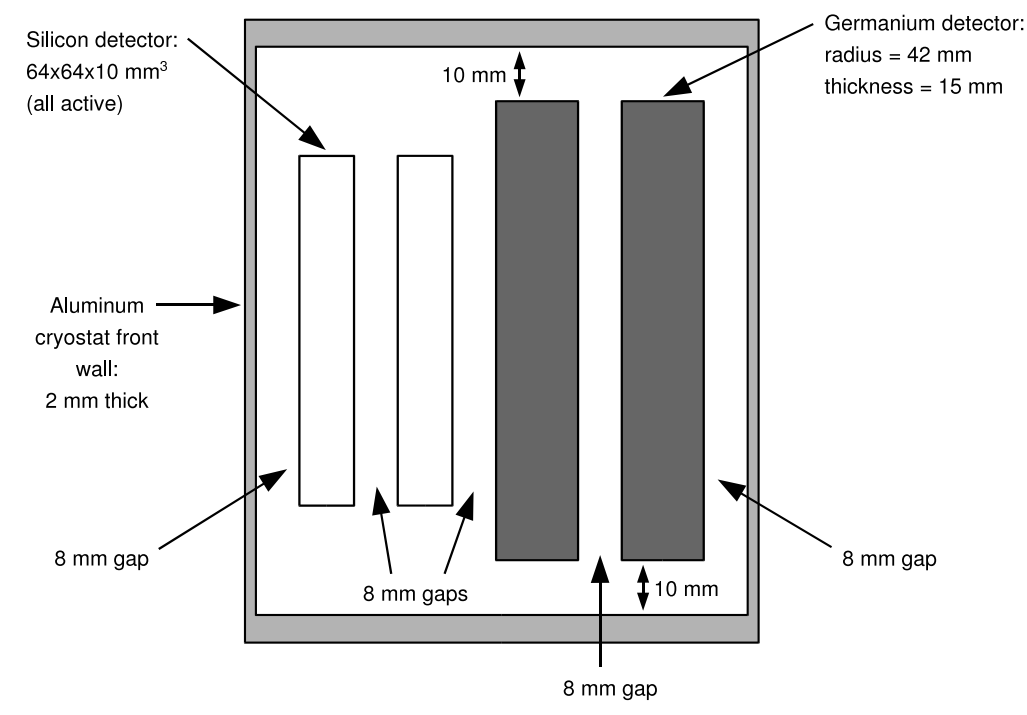

Si-Ge module

(front view)

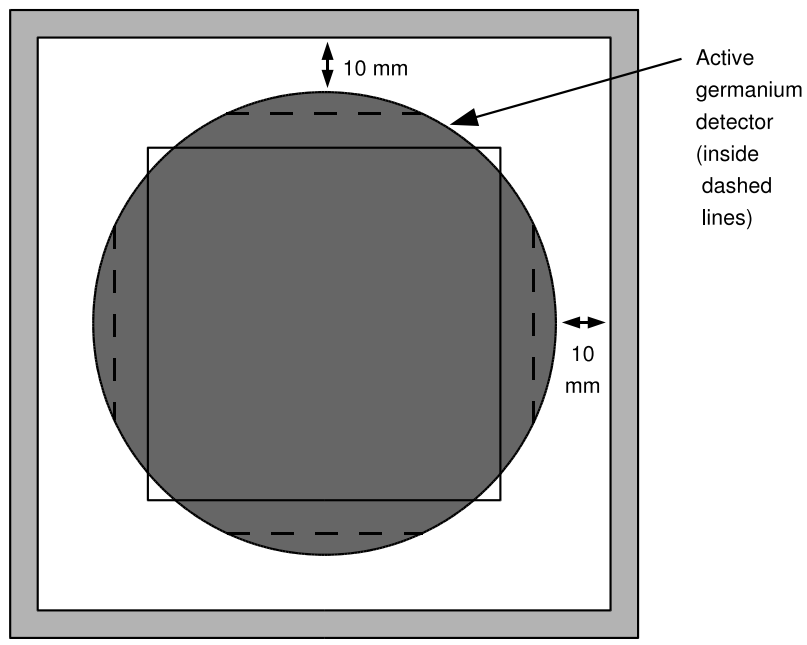

Figure 1: Simulated detector geometry for an Si-Ge module, shown in cross-sectional views. In the front view, the position of each $\mathrm{Si}$ detector is also indicated. 


\section{Si-CZT module \\ (side view)}

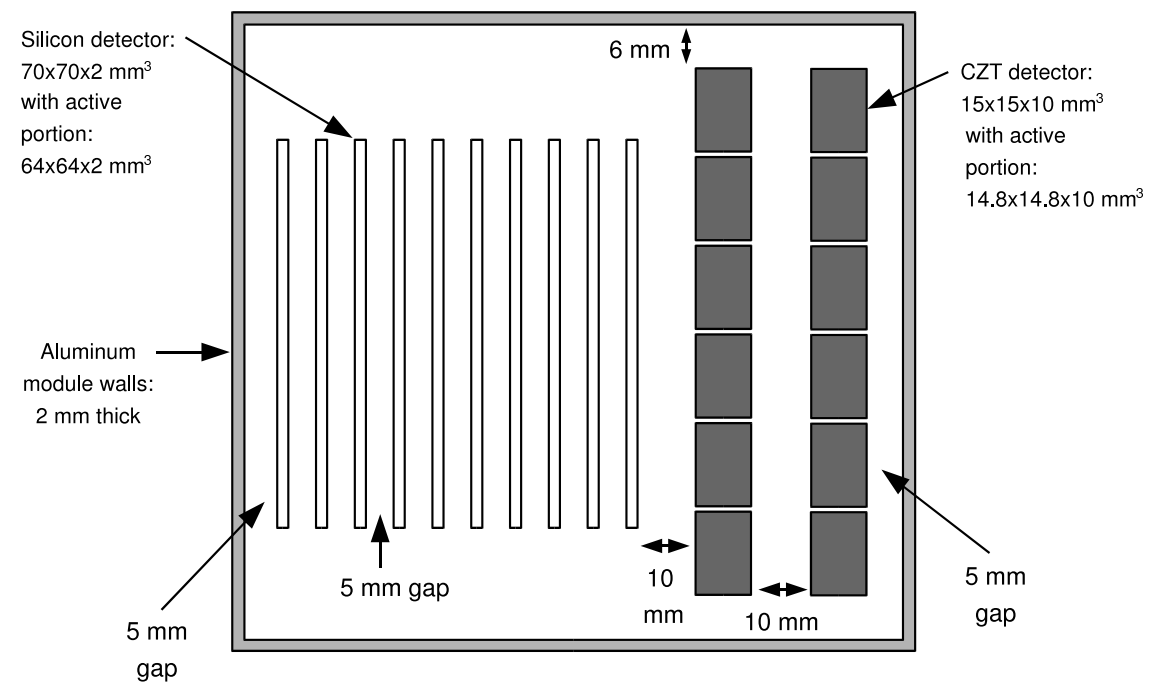

Si-CZT module

(front view)

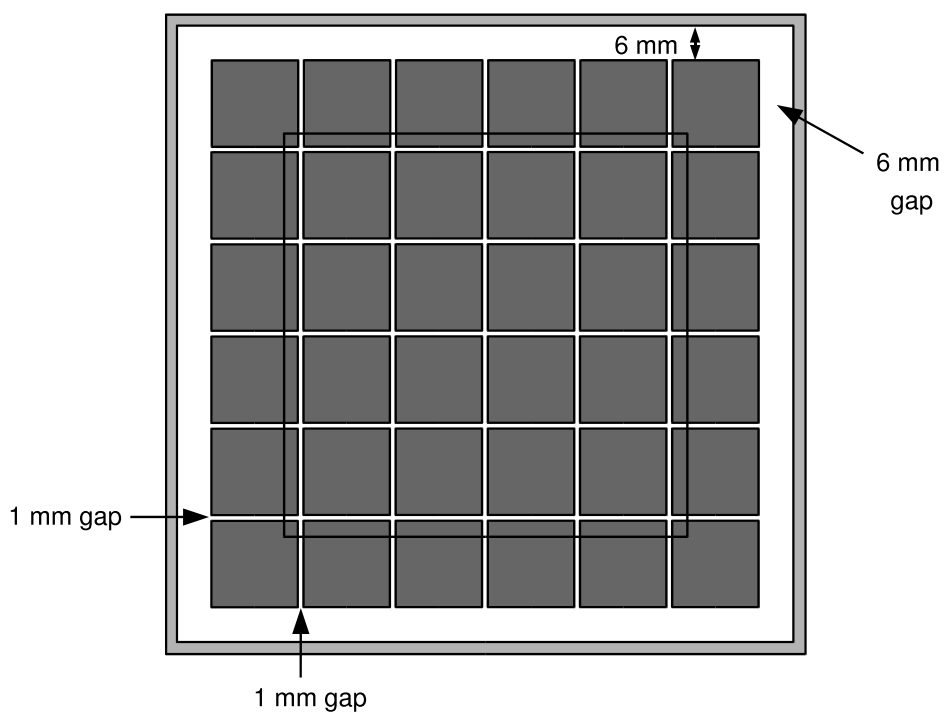

Figure 2: Simulated detector geometry for an Si-CZT module, shown in cross-sectional views. In the front view, the position of each Si detector is also indicated. 


\section{Large-scale Compton imager $16 \times 16$ array of detector modules}

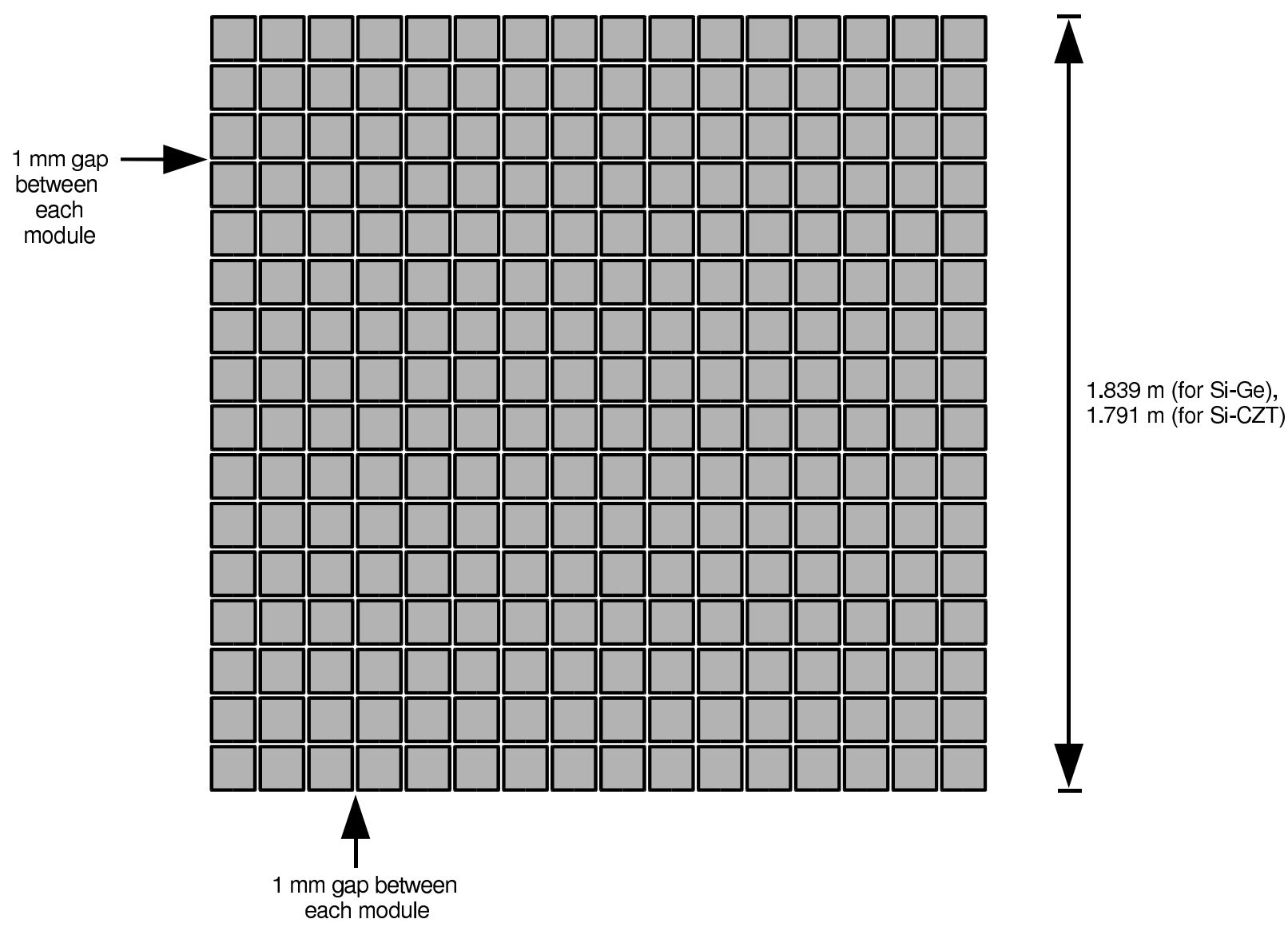

Figure 3: Simulated detector geometry for a large-scale Compton imager $16 \times 16$ array of detector modules. 


\begin{tabular}{ccc}
$\begin{array}{c}\text { Area contribution } \\
\text { in } 16 \times 16 \text { array }\end{array}$ & $\begin{array}{c}\text { Si-Ge } \\
\left(\mathrm{m}^{2}\right)\end{array}$ & $\begin{array}{c}\text { Si-CZT } \\
\left(\mathrm{m}^{2}\right)\end{array}$ \\
\hline Active $\mathrm{Si}$ & 1.05 & 1.05 \\
\hline Active Ge or active CZT & 1.32 & 2.02 \\
\hline $\begin{array}{c}\text { Active Ge or active CZT } \\
\text { behind active } \mathrm{Si}\end{array}$ & 1.04 & 0.90 \\
\hline Entire $16 \times 16$ array & 3.33 & 3.15 \\
\hline
\end{tabular}

Table 1: Area contributions for various detector components for both types of $16 \times 16$ array. The area of the entire $16 \times 16$ array is the area of the detector modules, not including the empty area due to the $1 \mathrm{~mm}$ gaps between each module.

\section{Energy resolution models}

To model the energy resolution for a detector, individual energy deposits are smeared using a Gaussian probability distribution with a specified FWHM at each energy.

All detector crystals in the Si-Ge detector have a FWHM energy resolution of $2 \mathrm{keV}$, for all energies. The energy resolution is worse for the $\mathrm{Si}-\mathrm{CZT}$ detector because the $\mathrm{Si}-\mathrm{Ge}$ detector is cooled, while the $\mathrm{Si}-\mathrm{CZT}$ detector operates at room temperature. In the $\mathrm{Si}-\mathrm{CZT}$ detector, the $\mathrm{Si}$ detector crystals have a FWHM energy resolution of $6 \mathrm{keV}$, for all energies. The energy resolution model used for the CZT detector crystals is based on measured CZT data, and is shown in Figure 4.

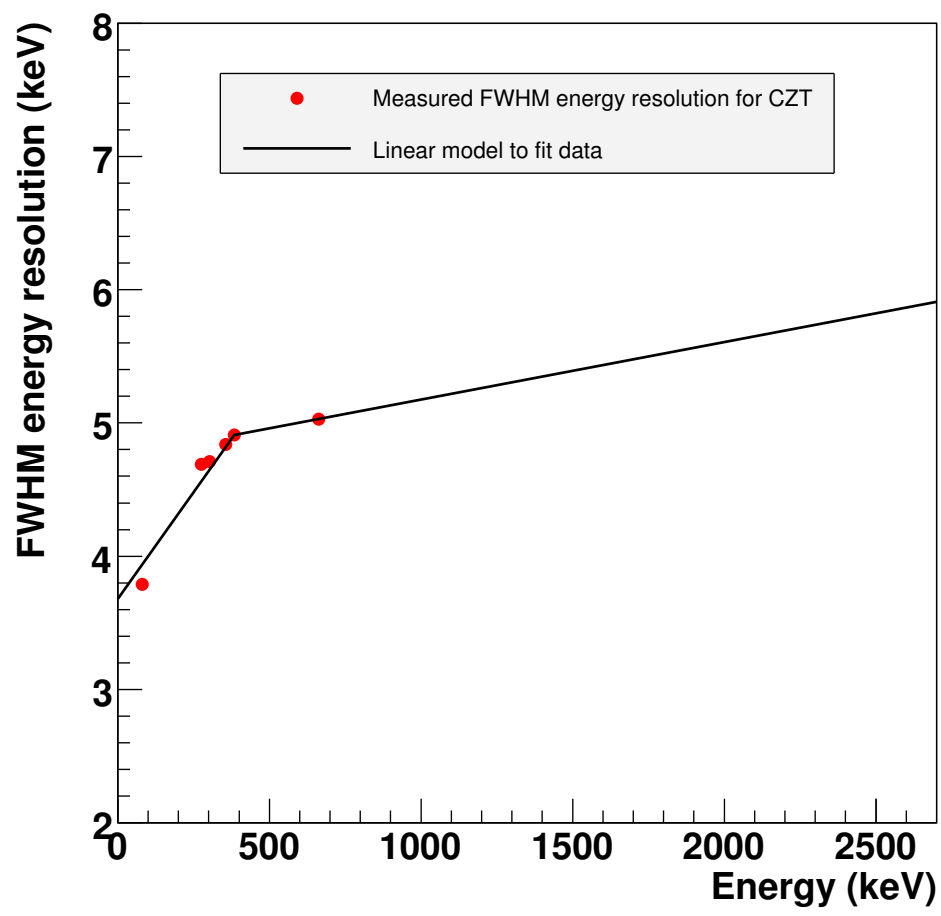

Figure 4: Energy resolution for CZT detector crystals. 


\section{Detector image resolution}

The detector image resolution is defined using the distance of closest approach (DOCA) of an image ring to the true source location. The Compton scattering angle of the first interaction and the positions of the first two interactions of an event define the opening angle and axis direction of a cone. This cone is backprojected onto a sphere which is centered at the detector. The intersection of the cone and the sphere is the Compton image ring, which is a ring-shaped curve that is nearly circular if the radius of the sphere is large compared to the detector length scale. Improving the energy resolution and the position resolution of interactions within the detector crystals improves the image resolution, because decreasing the uncertainties in interaction energies and positions decreases the amount by which an image ring is shifted away from the true source location.

We define the signal region size by specifying the signal region radius as the number of degrees along the signal DOCA distribution such that $85 \%$ of the total signal is contained within the signal region radius. We set the imaging bin solid angle equal to the signal region solid angle, and the total number of imaging bins is $4 \pi$ steradians divided by the solid angle of one imaging bin. The signal region size also determines the amount of background seen within the signal region. Improving the detector image resolution improves signal detection performance by increasing the number of imaging bins, and reducing the amount of background detected within the signal region.

Figure 5 shows simulated DOCA distributions for signal and background for the Si-Ge $16 \times 16$ array, for a $662 \mathrm{keV}$ point source located $100 \mathrm{~m}$ away from the detector array, on the center axis of the detector array, and $85 \%$ of the total signal is contained within a signal region radius of 5.5 degrees.

Table 2 and Table 3 show the signal region radius, number of imaging bins, and the percent of total background rings which cross the signal region, for each type of detector, for five different energies. For each energy, the source is a point source located $100 \mathrm{~m}$ away from the detector array, on the center axis of the detector array.

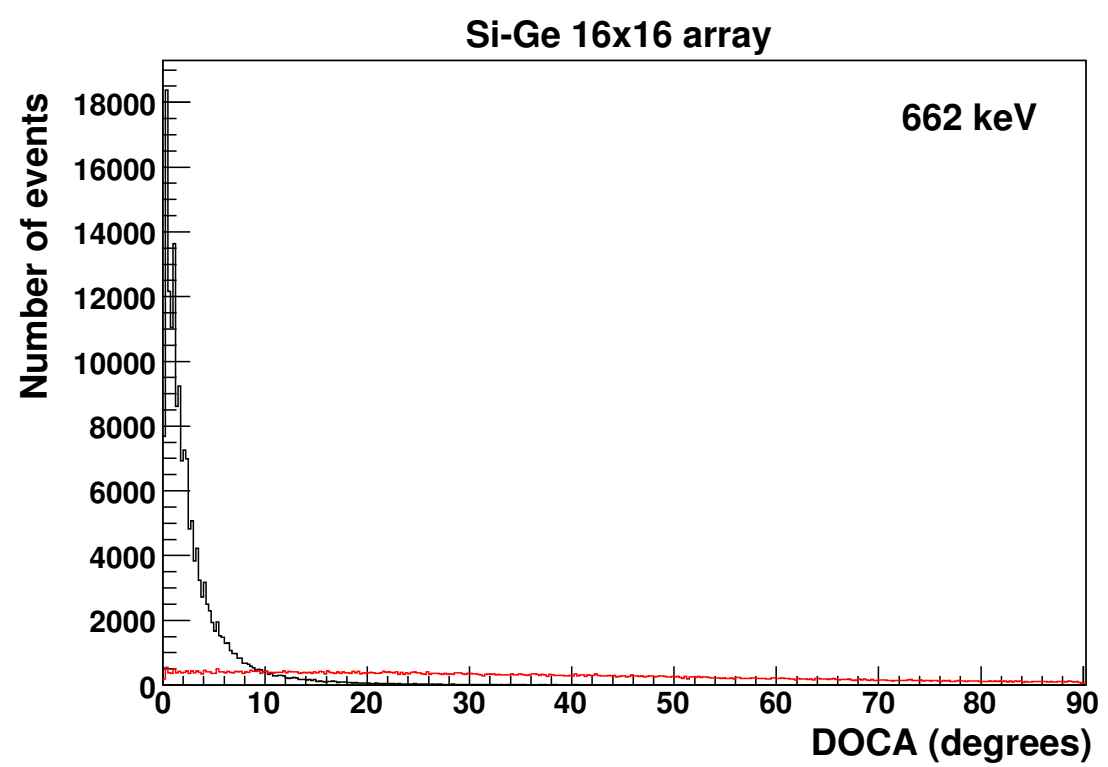

Figure 5: Simulated DOCA distributions for signal (black) and background (red) for the Si-Ge $16 \times 16$ array for a $662 \mathrm{keV}$ point source located $100 \mathrm{~m}$ away from the detector array, on the center axis of the detector array, and $85 \%$ of the total signal is contained within a signal region radius of 5.5 degrees. 


\begin{tabular}{cccc}
$\begin{array}{c}\text { Energy } \\
(\mathrm{keV})\end{array}$ & $\begin{array}{c}\text { Signal region } \\
\text { radius } \\
\text { (degrees) }\end{array}$ & $\begin{array}{c}\text { Number of } \\
\text { imaging bins }\end{array}$ & $\begin{array}{c}\text { Percent of total } \\
\text { background rings which } \\
\text { cross signal region }\end{array}$ \\
\hline 186 & 9.25 & 153 & $14.6 \%$ \\
414 & 6.5 & 311 & $9.4 \%$ \\
662 & 5.5 & 434 & $7.7 \%$ \\
1000 & 4.75 & 582 & $6.7 \%$ \\
2614 & 4.25 & 727 & $5.9 \%$ \\
\hline
\end{tabular}

Table 2: Si-Ge $16 \times 16$ array signal region radius, number of imaging bins, and the percent of total background rings which cross the signal region. For each energy, the source is a point source located $100 \mathrm{~m}$ away from the detector array, on the center axis of the detector array.

\begin{tabular}{cccc}
$\begin{array}{c}\text { Energy } \\
(\mathrm{keV})\end{array}$ & $\begin{array}{c}\text { Signal region } \\
\text { radius } \\
\text { (degrees) }\end{array}$ & $\begin{array}{c}\text { Number of } \\
\text { imaging bins }\end{array}$ & $\begin{array}{c}\text { Percent of total } \\
\text { background rings which } \\
\text { cross signal region }\end{array}$ \\
\hline 186 & 10.25 & 125 & $17.5 \%$ \\
414 & 7.75 & 218 & $11.4 \%$ \\
662 & 6.75 & 288 & $9.8 \%$ \\
1000 & 6.0 & 365 & $8.6 \%$ \\
2614 & 5.0 & 525 & $7.2 \%$ \\
\hline
\end{tabular}

Table 3: Si-CZT $16 \times 16$ array signal region radius, number of imaging bins, and the percent of total background rings which cross the signal region. For each energy, the source is a point source located $100 \mathrm{~m}$ away from the detector array, on the center axis of the detector array. 


\section{Detector imaging efficiency}

The imaging efficiency at a particular energy is defined as the number of events in the signal DOCA distribution within the signal region, divided by the total number of signal events incident upon the detector array at the particular energy. The signal region contains $85 \%$ of the total signal. Imaged events must pass the following event selection requirements. The event energy deposited in the detector crystals must be within a $\pm 10 \mathrm{keV}$ energy-cut window centered at the initial source photon energy. An event must produce at least two interactions in the detector crystals to be imaged. The first two interactions give the directional information of the incident photon. The total energy deposition for an event is the sum of all energy deposits for all interactions within the detector crystals. If the first two interactions for an event occur within the same pixel, then that event is rejected. If the first two interactions for an event are both within the same detector crystal, then the two interactions must be separated by at least 4 pixel widths. Table 4 shows the imaging efficiencies for both types of $16 \times 16$ array, for five different energies.

\begin{tabular}{ccc}
$\begin{array}{c}\text { Energy } \\
(\mathrm{keV})\end{array}$ & $\begin{array}{c}\text { Si-Ge } \\
\text { imaging } \\
\text { efficiency }\end{array}$ & $\begin{array}{c}\text { Si-CZT } \\
\text { imaging } \\
\text { efficiency }\end{array}$ \\
\hline 186 & $6.1 \%$ & $7.5 \%$ \\
414 & $4.9 \%$ & $9.7 \%$ \\
662 & $3.6 \%$ & $7.6 \%$ \\
1000 & $2.8 \%$ & $5.7 \%$ \\
2614 & $1.3 \%$ & $2.5 \%$ \\
\hline
\end{tabular}

Table 4: Imaging efficiencies for both types of $16 \times 16$ array.

\section{Receiver Operating Characteristics (ROC) curves}

We calculate our ROC curves analytically [1] using Poisson probability distributions for the detected background count rate and the detected signal-plus-background count rate. Each point along a ROC curve corresponds to a different detection threshold. During the measurement time, if the number of background counts fluctuates above the detection threshold, then the background fluctuation looks like a source (generating a false alarm). We numerically integrate the Poisson distributions for the background count rate and the signal-plus-background count rate, to determine the false detection probability and the detection probability. Since a false alarm can come from any of the spatial imaging bins, we report the false-alarm rate as the total number of false alarms per unit time. Our analytic ROC curve calculations take the following ten input parameters:

1. Measurement time.

2. Detector area.

3. Detector altitude.

4. Imaging efficiency.

5. Source emission rate. 
6. Signal photon energy.

7. Air attenuation of signal photons.

8. Background photon flux into the detector as a function of energy, within a $\pm 10 \mathrm{keV}$ energy-cut window. Effects of air attenuation and scattering are included.

9. Signal region size (image resolution).

10. The number of background rings which cross the signal region ("ring effect").

We define the signal region size by specifying the signal region radius as the number of degrees along the signal DOCA distribution such that $85 \%$ of the total signal is contained within the signal region radius. We set the imaging bin size to correspond to the signal region solid angle. Since the background photons are imaged as rings, not points, background events which are strictly outside the signal region produce rings which can still cross inside the signal region. This "ring effect" [1] causes the Compton imaging detector to see more background in the signal region than it would if it detected background events as points which were uniformly distributed throughout $4 \pi$ steradians. The amount of "ring effect" is determined by the fraction of the total number of background rings which cross the signal region. Each of the imaging bins contains background, so every imaging bin has the possibility of producing a false alarm.

Table 5 shows the percent of the initial signal photon flux reaching the detector after air attenuation through a distance of $100 \mathrm{~m}$, and the background photon flux at an altitude of $100 \mathrm{~m}$, for five different energies. The simulations to determine the air attenuation and background photon flux [1] were performed using GEANT4.

\begin{tabular}{ccc} 
& $\begin{array}{c}\text { Percent of initial } \\
\text { signal photon flux } \\
\text { reaching detector after } \\
\text { air attenuation through } \\
\text { distance of } 100 \mathrm{~m}\end{array}$ & $\begin{array}{c}\text { Background photon flux } \\
\text { at altitude of } 100 \mathrm{~m} \\
\left.\text { (photons } / \mathrm{m}^{2} / \mathrm{sec}\right)\end{array}$ \\
\hline 186 & $23.4 \%$ & 323 \\
414 & $31.6 \%$ & 109 \\
662 & $37.8 \%$ & 55 \\
1000 & $43.7 \%$ & 27 \\
2614 & $61.8 \%$ & 302 \\
\hline
\end{tabular}

Table 5: Percent of initial signal photon flux reaching the detector after air attenuation through a distance of $100 \mathrm{~m}$, and background photon flux at an altitude of $100 \mathrm{~m}$, for five different energies. The simulations to determine the air attenuation and background photon flux were performed using GEANT4. 


\section{Results}

Figure 6 and Figure 7 show ROC curves for each type of $16 \times 16$ array, for a ${ }^{137}$ Cs source, and each plot shows a ROC curve which passes through $95 \%$ detection probability at one false alarm per hour, and a ROC curve which passes through $50 \%$ detection probability at one false alarm per hour. Each plot is for a measurement time of one minute, and a detector altitude of $100 \mathrm{~m}$. These results include the fact that the branching ratio is $85 \%$ for a ${ }^{137} \mathrm{Cs}$ decay to emit a $662 \mathrm{keV}$ photon. A source strength for each ROC curve is indicated on each plot.

Table 6 shows detected source strengths in units of emitted source photons/second, for both types of $16 \times 16$ array, for $95 \%$ detection probability, for one false alarm per hour, for a measurement time of one minute, for a detector altitude of $100 \mathrm{~m}$, for five different energies. Source photons are emitted isotropically into $4 \pi$ steradians. The detected source strengths were determined using the ROC curve calculations.

Table 7 shows detected source strengths in units of emitted source photons/second, for both types of $16 \times 16$ array, for $50 \%$ detection probability, for one false alarm per hour, for a measurement time of one minute, for a detector altitude of $100 \mathrm{~m}$, for five different energies. Source photons are emitted isotropically into $4 \pi$ steradians. The detected source strengths were determined using the ROC curve calculations.

The performance of the two different detectors can be compared by comparing their detected source strengths. The background is high at $2614 \mathrm{keV}$, so the $2614 \mathrm{keV}$ results have larger detected source strengths than at $1000 \mathrm{keV}$. All results are for a point source located $100 \mathrm{~m}$ below the detector array, on the center axis of the detector array. The detection performance of the Si-CZT detector is found to be better than the $\mathrm{Si}-\mathrm{Ge}$ detector. This is primarily because the $\mathrm{Si}-\mathrm{CZT}$ detector has better imaging efficiency than the $\mathrm{Si}-\mathrm{Ge}$ detector.

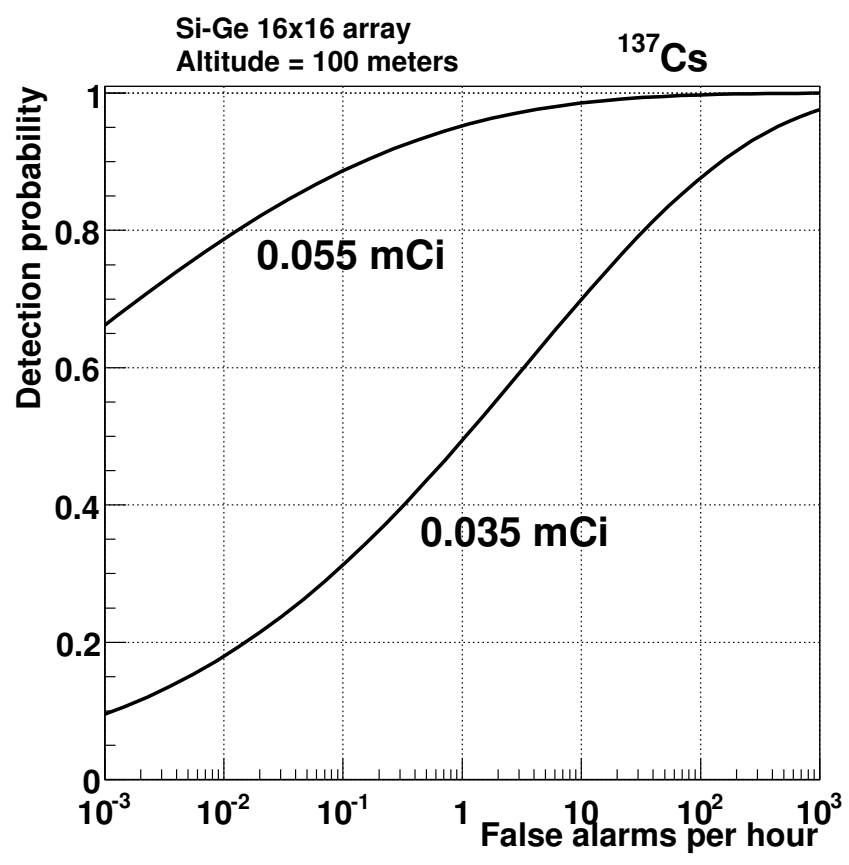

Figure 6: ROC curves for the Si-Ge $16 \times 16$ array, for a ${ }^{137} \mathrm{Cs}$ source, with an energy-cut window of $662 \pm 10 \mathrm{keV}$, with a measurement time of one minute, and a detector altitude of $100 \mathrm{~m}$. These results include the fact that the branching ratio is $85 \%$ for a ${ }^{137} \mathrm{Cs}$ decay to emit a $662 \mathrm{keV}$ photon. 


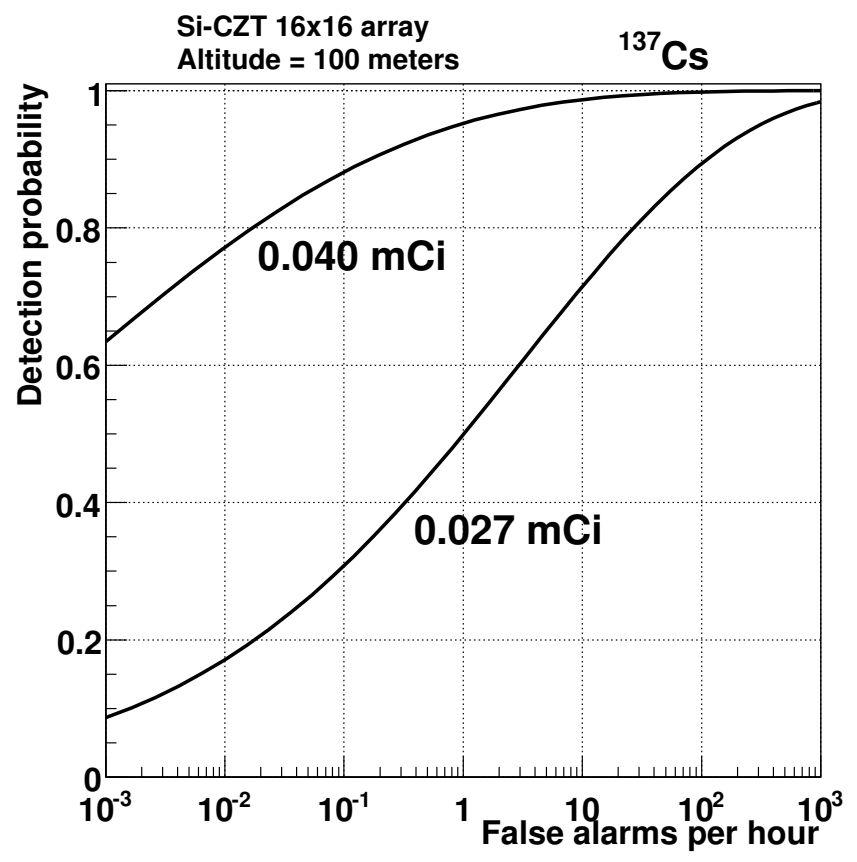

Figure 7: ROC curves for the Si-CZT $16 \times 16$ array, for a ${ }^{137} \mathrm{Cs}$ source, with an energy-cut window of $662 \pm 10 \mathrm{keV}$, with a measurement time of one minute, and a detector altitude of $100 \mathrm{~m}$. These results include the fact that the branching ratio is $85 \%$ for a ${ }^{137} \mathrm{Cs}$ decay to emit a $662 \mathrm{keV}$ photon.

\begin{tabular}{ccc}
$\begin{array}{c}\text { Energy } \\
(\mathrm{keV})\end{array}$ & $\begin{array}{c}\text { Si-Ge } \\
\text { (photons/second) }\end{array}$ & $\begin{array}{c}\text { Si-CZT } \\
\text { (photons/second) }\end{array}$ \\
\hline 186 & $5.92 \times 10^{6}$ & $5.88 \times 10^{6}$ \\
414 & $2.52 \times 10^{6}$ & $1.92 \times 10^{6}$ \\
662 & $1.74 \times 10^{6}$ & $1.26 \times 10^{6}$ \\
1000 & $1.26 \times 10^{6}$ & $9.25 \times 10^{5}$ \\
2614 & $3.59 \times 10^{6}$ & $2.78 \times 10^{6}$ \\
\hline
\end{tabular}

Table 6: Detected source strengths in units of emitted source photons/second, for both types of $16 \times 16$ array, for $95 \%$ detection probability, for one false alarm per hour, for a measurement time of one minute, for a detector altitude of $100 \mathrm{~m}$, for five different energies. Source photons are emitted isotropically into $4 \pi$ steradians. 


\begin{tabular}{ccc}
$\begin{array}{c}\text { Energy } \\
(\mathrm{keV})\end{array}$ & $\begin{array}{c}\text { Si-Ge } \\
(\text { photons/second })\end{array}$ & $\begin{array}{c}\text { Si-CZT } \\
\text { (photons/second) }\end{array}$ \\
\hline 186 & $4.03 \times 10^{6}$ & $4.00 \times 10^{6}$ \\
414 & $1.67 \times 10^{6}$ & $1.30 \times 10^{6}$ \\
662 & $1.11 \times 10^{6}$ & $8.51 \times 10^{5}$ \\
1000 & $7.77 \times 10^{5}$ & $5.92 \times 10^{5}$ \\
2614 & $2.37 \times 10^{6}$ & $1.85 \times 10^{6}$ \\
\hline
\end{tabular}

Table 7: Detected source strengths in units of emitted source photons/second, for both types of $16 \times 16$ array, for $50 \%$ detection probability, for one false alarm per hour, for a measurement time of one minute, for a detector altitude of $100 \mathrm{~m}$, for five different energies. Source photons are emitted isotropically into $4 \pi$ steradians.

\section{References}

[1] D. Lange, H. Manini, D. Wright, Large-Scale Compton Imaging for Wide-Area Surveillance, UCRL-TR-219458. 